\title{
Information processing and concomitant heart rate changes in the overweight and underweight
}

\author{
J. RICHARD JENNINGS \\ Walter Reed Army Institute of Research, Department of Experimental Psychophysiology, \\ Forest Glen Section, Building 189, Walter Reed Army Institute of Research, \\ Walter Reed Army Medical Center, Washington, D. C. 20012
}

\begin{abstract}
Two possible interpretations of the Laceys' hypothesized relationship between cardiac acceleration and "mental elaboration" were explored in 24 overweight and 24 underweight individuals. The relative contribution of cognitive manipulation and memory requirements to cardiac change was investigated in five tasks. Several aspects of the results suggested that memory requirements were related more closely than cognitive manipulation to cardiac changes during task performance. However, since rankings of the tasks in terms of both memory load and energy demands were significantly related to the cardiac changes, a single factor interpretation of the cardiac effects was not justified. Body weight effects for both cardiac deceleration and memory errors were dependent upon the difficulty of the tasks. Relative to the underweight, the overweight showed greater deceleration with low difficulty and more errors with high difficulty items. The results suggest a relationship between heart rate change and memory demands and a limitation upon the generality of Schachter's concept of sensory binding in the obese.
\end{abstract}

The primary aim of the current study was the investigation of concomitant changes in heart rate and cognitive processes. Cardiac acceleration has been related to rejection of external input and mental elaboration by the Laceys' (Lacey, Kagan, Lacey, \& Moss, 1963; Lacey, 1967; Lacey, B. \& Lacey, J., 1974). The experimental support for this notion has been questioned, however, due to the frequent confounding of stimulus variables and response requirements with cognitive variables (Elliott, 1972; Hahn, 1973). The present experiment employed a single set of visual stimuli and systematically varied the cognitive processes required of the subject. Response requirements were essentially the same for all tasks. Thus, the design permitted differences in the direction and magnitude of cardiac response to be associated with differences in cognitive processes, rather than with differences in stimulus or response characteristics. The cognitive processes required of the subject were varied along broad rather than narrow functional lines. Thus, tasks which required active memory or rehearsal were compared to those which required cognitive transformations or manipulations, instead of comparing the effects of specific variables within a single memory task. This design necessarily sacrificed a degree of explicitness in order to explore more than one cognitive function. Further explicitness may be achieved, however, by parametric follow-up experiments once a broad cognitive function is implicated by an initial experiment. In effect, the

The assistance of Kay Jennings, James Struthers, Jeanne Stringfellow, and Charles C. Wood at various phases of this study is gratefully acknowledged. Portions of the results were presented at the 1973 meeting of the Society for Psychophysiological Research. present experiment attempted to provide a bridge between the global concept of "mental elaboration" and more detailed concepts of cognitive functioning.

A second aim of the study was to explore the relation of heart rate responses to possible differences in information processing between the overweight and underweight. Schachter (1971) has suggested that the overweight are more responsive than normal individuals to external stimuli above some threshold. Thus, Schachter has generalized his previous notion that eating in the obese is controlled predominately by external cues. Rodin (1973) found some support for a generalized "sensory binding" concept in overweight subjects using simple information processing tasks. If the overweight can be shown to process information differently from underweight subjects, then different weight groups provide a variation in processing that may assist in understanding the potential sensitivity of heart rate to different cognitive processes. By dividing the current tasks into a temporal period associated with initial information input and storage and a period for performance of the specific task, the current design provided an interval in which "sensory binding" in the overweight might occur fairly clearly. The relationships between task performance and cardiac responses (both acceleration and deceleration) could then be examined in the context of differences between weight groups during both information input and task performance periods. Two levels of task difficulty were employed to test the generality of possible effects of the task or body weight manipulations.

\section{METHOD}

\section{Subjects}

Forty-eight male college students between 18 and 29 years of age served as paid volunteers. Subjects were selected on the basis of their body weight in relation to their height and build: those 
$5 \mathrm{lb}$ or more above the maximum desirable weight from Metropolitan Life Insurance tables (1959) were placed in an overweight group, while those less than the minimum desirable weight were placed in the underweight group. The mean weight-height (pounds/inches) ratio for the overweight group was $2.77, \mathrm{SD}=.36$; for the underweight group $1.96, \mathrm{SD}=.22$.

\section{Tasks}

Each task was initiated by the appearance of an "information" slide, which was present for $5 \mathrm{sec}$. This slide presented six numbers displayed in two columns. Five different tasks could be assigned immediately after the information slide. The specific task assigned on each trial was randomly determined. The slide assigning the task always contained a brief instruction, e.g., add; but some slides repeated the numbers from the information slide while others did not. The five tasks presented below are labeled by the instruction included on the task slides.

(1) Read silently. This slide, which included the numbers repeated from the information period, was present for $5 \mathrm{sec}$, after which the subject wrote down the last number read from the slide. This task was assumed to require attention to the visual input but required little if any cognitive manipulation or active memory load.

(2) Store. Subjects were required to remember the numbers from the information slide for $5 \mathrm{sec}$ and then record them in proper serial order. Relative to the read task, this task placed an increased memory load on the subject. Only a minimal amount cognitive manipulation was assumed to be required by operations such as rehearsal and association.

(3) Add. The six numbers from the information slide were repeated and subjects added the numbers and recorded the sum. Cognitive manipulation was required by the addition, but the memory requirement was limited to the storage of intermediate and final sums.

(4) Add from memory. Subjects computed from memory the sum of the numbers presented during the information period. Memory for the numerical input and partial and final sums was required. Cognitive manipulation of the same type as in the add task was assumed to be required.

(5) Rule. This instruction was presented with the numbers from the information slide. Subjects were required to identify the arithmetic rule relating numbers in the left and right columns of the display. This task was assumed to require memory for past rules applied if the correct solution was not immediate, and it required greater cognitive manipulation than the two add tasks.

Each subject received 50 trials, 10 of each task, with task order randomized. Four or five training trials on each task were given prior to the 50 trials. A trial was composed of a variable intertrial interval (mean of $23 \mathrm{sec}$, range of 17 to $32 \mathrm{sec}$ ), followed by an unsignaled 10-sec pre period, the 5-sec information period, and, finally, the operate period during which the task was performed.

Based on the assumptions listed above, the tasks were ranked in terms of cognitive manipulation and memory requirements. These ranks were generated after initial data analysis suggested the value of obtaining quantitative estimates of the strength of relationship between the different requirements and cardiac change. Thus, the ranks were developed for regression analyses performed with the cardiac data. Tied ranks were assigned when relatively clear order judgment seemed impossible. The ranks from low to high for cognitive manipulation were: 1 . Read, 2. Store, 3.5 Add, 3.5 Add-Memory, and 5. Rule; for memory requirements: 1. Read, 2.5 Rule, $2.5 \mathrm{Add}, 4$. Store, and 5. Add-Memory. A second set of ranks based on subjective aspects of the tasks were obtained directly from the subjects following the experiment. Subjects assigned numerical ranks to the different tasks according to the degree of relative difficulty, interest, frustration, and energy demand.
The information slides were created at two difficulty levels as defined by the uncertainty measure of information theory. Random sampling from the numbers 0 to 20 produced low uncertainty slides and from the numbers 0 to 35 produced high uncertainty. Two constraints were placed on the sampling: first, the same number could appear only once on a slide of six numbers; and second, the slides for the rule task had to show the requisite arithmetic constraint. With respect to the rule task, six of the transformations used were simple one-step operations, e.g., $y=x+8$. Four were two-step operations, e.g., $y=3 x-1$. Prior to the experimental session, subjects were told what range of numbers to expect and also what types of arithmetic transforms could be used in the rule task.

\section{Equipment and Electrode Placements}

The stimuli were black and white $20 \times 30 \mathrm{~cm}$ images projected from a Kodak Carousel projector onto a rear projection screen. Subjects sat about $1 \mathrm{~m}$ from the screen. Slide presentation and timing were controlled electronically except for the termination of the operate period which was controlled by the subject's response press or an experimenter response switch. 1

Silver-silver chloride plated electrodes with Beckman paste were used for the cardiac measures. Signals were acquired with a Grass Model 5 polygraph. The cardiac signal was processed to produce a pulse coincident with the $\mathrm{R}$ wave. For the first 24 subjects, the interval between these pulses was sensed electronically and printed on a Hewlett-Packard Model 5050b printer. For the remaining subjects, the signal was recorded on an Ampex 2000 series audio tape recorder and subsequently read into a General Automation 1830 computer which detected and recorded the interbeat interval, IBI.

\section{Experimental Design}

The basic design of the experiment was a three-way analysis of variance design with repeated measurement of one factor (Winer, 1962, Chapter 7). The repeated measures factor was the five tasks, i.e., all subjects performed all tasks. Nonrepeated factors were obesity and uncertainty. Twelve subjects were in each of the four independent groups: obese/high uncertainty, obese/low uncertainty, underweight/high uncertainty, and underweight/low uncertainty.

\section{Data Reduction}

Task performance was evaluated using number of errors calculated for each of the five tasks. Latency of response was measured for the add, add-memory, and rule tasks in which the subject determined the length of the operate period.

The IBI data were converted to mean IBI per second for each second during the experimental periods (Jennings, Strinfellow, \& Graham, 1974; Khachturian, Kerr, Kruger, \& Schachter, 1972). Each subject's IBI data were considered separately for each task. The 10 trials of each task were assumed to incorporate the same set of psychological processes regardless of correctness of task performance, and IBI scores were averaged across the 10 trials. These averaged "cardiac evoked responses" for each subject were the basic cardiac data. Initial analyses on the maximum, minimum, and mean IBI for the pre, information, and operate periods established that consistent changes in IBI across subjects were associated with these periods and justified the use of difference scores as summary indices reflecting the "cardiac evoked response."

Difference scores served to adjust for the subject's baseline IBI and were capable of representing specific features of somewhat idiosy ncratic individual cardiac response curves. The maximum and minimum within each of the trial periods were subtracted from the pre period mean IBI to form the difference scores. The mean IBI during the pre period was not significantly related to the weight, uncertainty, or task variables. However, many subjects did show a slight cardiac deceleration at the end of the 
pre period immediately prior to the onset of the information slide. (Sign test of differences between Seconds 3 and 8, ratio $=32 / 44 ; Z=5.88)$. In cases where difference scores were correlated with pre period average or difference scores, analysis of covariance was applied. Analysis of the covaried scores showed the same patterns of significant results found using the unadjusted scores.

Two types of difference score were defined for each period, an acceleration score and a deceleration score. For example, the deceleration score for the operate period was defined as the maximum for the operate period minus the mean for the pre period. Similarly, the acceleration score for the information period was defined as the minimum for the information period minus the mean for the pre period. Similar acceleration and deceleration scores were computed for each period in each task. ${ }^{2}$ The score names were placed in terminology appropriate for changes in heart rate even though the differences were in IBI, the reciprocal of rate. This terminology was adopted in order to facilitate a comparison with previous work on the Lacey hypothesis.

\section{RESULTS}

Cardiac responses during the information input periods will be presented first, followed by the results for each of the three main variables: tasks, uncertainty or difficulty, and body weight. Post-hoc tests were performed using the Tukey (b) procedure (Winer, 1962, p. 87) for main effects and Cichetti technique (1972) for interaction effects. A rejection region of .05 was adopted. When appropriate, degrees of freedom for within subject comparisons were reduced (Box correction, See Davison, 1972).

\section{Cardiac IBI During the Information Periods}

The cardiac reaction during the information input was a stable acceleration relative to the pre period baseline (see response curves in Figure 2). Virtually all subjects showed an accelerative change during this period. As shown later, however, some subjects showed brief decelerative changes before or after a more general episode of cardiac acceleration. The statistical significance of the difference between the pre period and information input period cardiac IBI was established by analyses of the mean, maximum, and minimum across the periods. In all analyses, the main effect of period was significant (all $F s \geqslant 5.18$, adjusted $\mathrm{df}=1,88$ ). According to post-hoc tests, the information period produced a decrease in both the mean and maximum IBI relative to the pre period.

\section{Tasks}

As shown in Figure 1, the cardiac responses differed between tasks. However, these differences did not seem to be a direct function of either the hypothetical demands for cognitive manipulation or memory. The differences between the cardiac responses for the different tasks were verified by analyses of variance on the cardiac difference scores from the operate period. Significant main effects of tasks were found for both the

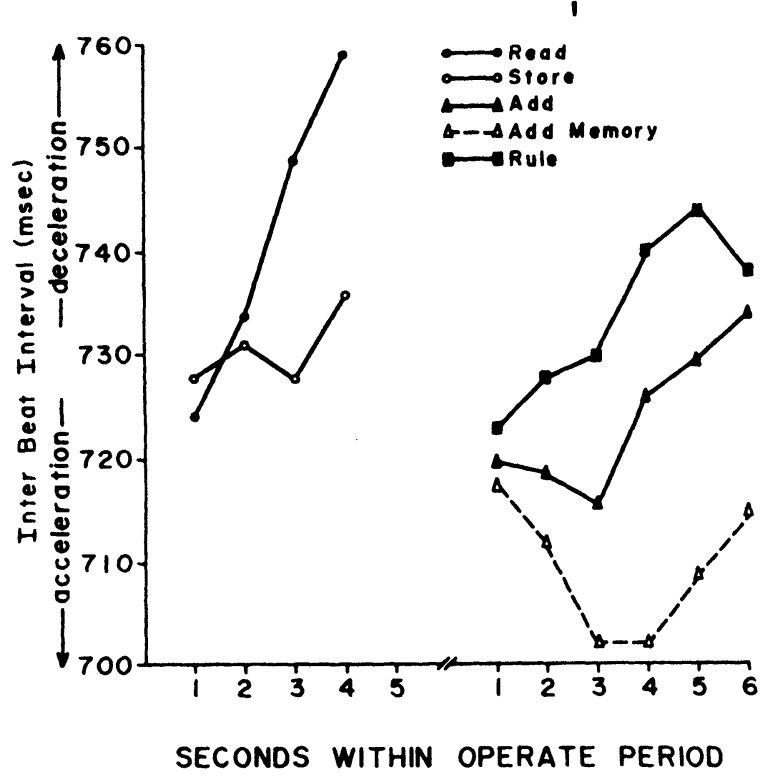

Figure 1. Operate period IBI response curves plotted separately for the five tasks. Curves for the read and store task are shown in the left portion while those for the add, add-memory, and rule tasks are shown on the right. Means for the pre period and information input period were 740 and 725 msec.

acceleration $(\mathrm{F}=9.61$, adjusted $\mathrm{df}=1,176)$ and deceleration scores $(F=4.74)$. The deceleration scores for the read and rule tasks were significantly larger than those for the store and add-memory tasks. The remaining comparisons within the deceleration scores were not significant. The acceleration scores for add-memory were significantly greater than those for all of the other tasks; those for add were significantly greater than those for read. Thus, acceleration was present primarily in the two add tasks, while deceleration appeared with tasks having external input and disappeared when memory requirements were present.

All of the postexperimental ratings (relative difficulty, interest, energy requirement, and frustration) produced similar orderings of tasks which were consistent across subjects (Friedman analysis of variance: $\chi^{2}(4) \geqslant 80.3$ ). The average energy demand ratings, for example, ordered the tasks from least to most: read, add, store, add-memory, and rule.

The average cardiac changes presented above reflect only the trends which are consistent across all subjects. As an attempt to analyze the task effects on the IBI data without obscuring individual response patterns, each individual's cardiac results were compared with the rank orders of the tasks based on the cognitive manipulation, memory requirements, and energy demands by means of regression analyses. The average of these within subject regression weights was tested for significance using a 


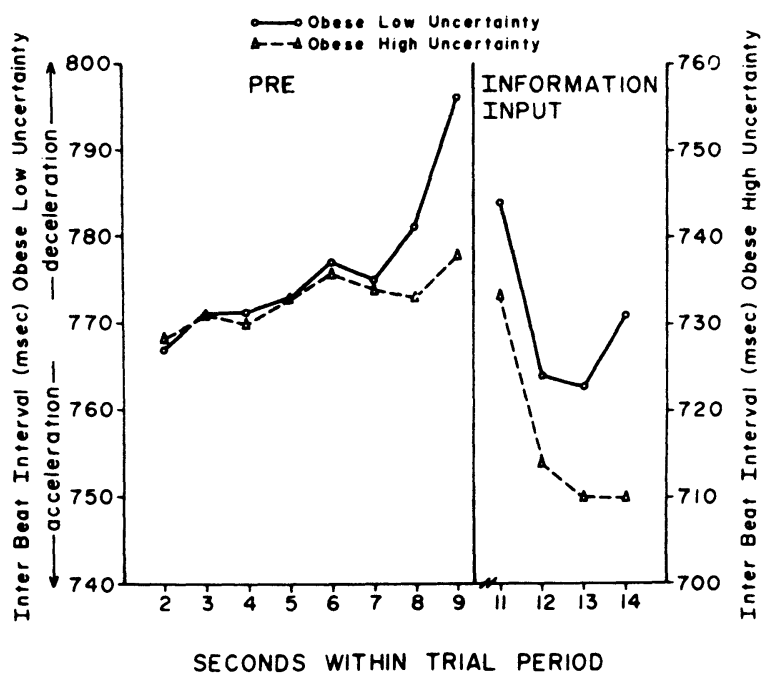

Figure 2. Pre and information input period IBI response curves plotted separately for the obese (overweight) low uncertainty and obese (overweight) high uncertainty groups. The two groups are scaled separately due to differences in baseline IBI. The scale for the obese low uncertainty group is on the left ordinate; for the obese high-uncertainty, it is on the right ordinate.

$\mathrm{t}$ test with $\mathrm{df}=47$. Memory ranks produced a significant Beta of $6.4(\mathrm{t}=4.2)$ for acceleration and $-5.5(\mathrm{t}=-3.8)$ for deceleration; however, the cognitive manipulation ranks did not produce significant Beta weights. Betas between both cardiac scores and energy demand ratings were significant (Acceleration Beta $=5.5, \mathrm{t}=5.2$; Deceleration Beta $=-3.7, \mathrm{t}=-2.1)$.

A direct comparison of the strength of relationship between cardiac change and ranks based on memory demand, cognitive manipulation, and energy demand was made with dependent $t$ tests. Betas for energy demand were higher than those for cognitive manipulation within both acceleration $(\mathrm{t}=-2.12$, $\mathrm{df}=46)$ and deceleration scores $(\mathrm{t}=-2.11$, $\mathrm{df}=46)$. Betas for the memory ranks were higher than those for cognitive manipulation only within the deceleration scores $(t=3.57, d f=47)$. Betas for memory and energy demand ranks did not differ significantly. Thus, based on the within subject regression weight comparisons, cognitive manipulation ranks were generally less predictive of change in IBI than either memory or energy demand ranks.

\section{Uncertainty}

Despite clear increases in performance errors with high uncertainty, task-related changes in cardiac IBI were not significantly influenced by uncertainty or difficulty per se. As noted below, however, uncertainty did interact with other variables.

\section{Body Weight}

According to Schachter's hypothesis, sensory binding and internal information processing might be expected to be incompatible. Thus, overweight subjects were expected to perform relatively poorly on tasks requiring processing in the presence of sensory input, i.e., add and rule, but relatively well on tasks requiring only processing of stored information, i.e., store and add-memory. These expectations were partially confirmed; however, the results of the body weight variable were dependent upon uncertainty level.

The influence of body weight on performance was seen primarily in the tasks requiring memory processing, i.e., store and add-memory. While the overweight and underweight subjects did not differ in average errors across tasks, the Body Weight by Uncertainty by Task interaction was significant $[\mathrm{F}(3,132)=2.78]$. This interaction was due primarily to the effect of obesity and uncertainty on performance of the store task. Within the add from memory task, errors were not associated with body weight; however, obese subjects showed a shorter log latency than underweight subjects [1.12 vs. $1.19 \log$ seconds, $p<.05$ post-hoc test based on Weight by Task interaction, $F(2,88)=3.48]$.

The relation of body weight and uncertainty was studied more closely within the store task using an error score reflecting errors of both order and omission. ${ }^{3}$ These memory scores showed a significant Body Weight by Uncertainty interaction $[F(1,44)=6.24]$ when analyzed with a two-way factorial analysis of variance. Obese subjects made more errors than underweight subjects with the high-certainty input. With low uncertainty input, errors did not significantly differ between obese and underweight subjects. Schachter's expectation that the overweight would perform better than underweight was supported marginally by the direction of the insignificant difference between the means within the low uncertainty condition.

Given the presence of some performance differences, cardiac differences between weight groups during information input and initial storage and task performance were of particular interest. Arguing loosely from the Lacey hypothesis, "sensory binding" might be expected to produce decelerative changes in the obese. This was true only in the sense that overweight subjects showed less acceleration than the underweight. Decelerative changes in an absolute sense were minimal in the average data. In a relative sense, however, the decelerative scores were larger in the overweight than in underweight during both information input $[F(1,44)=13.56]$ and task performance $[\mathrm{F}(1,44)=5.38]$.

The decelerative changes in the information period were a function of uncertainty as well as body weight [Uncertainty by Body Weight, $F(1,44)=7.26$ ]. Figure 2 compares the second-by-second IBI curves for the 

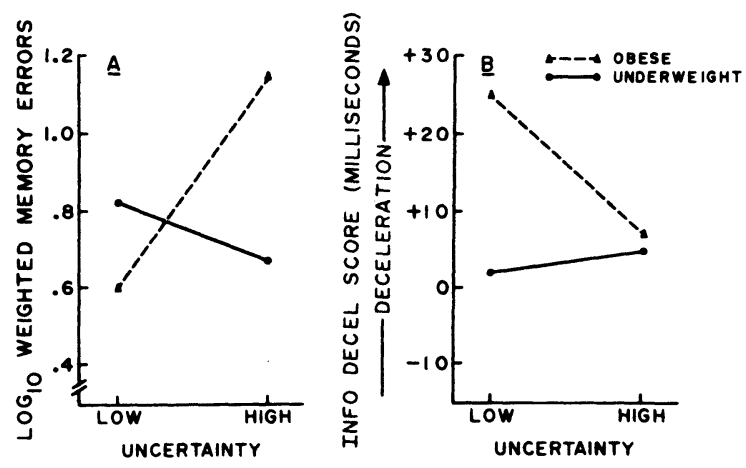

Figure 3. Parallel interactions of performance and cardiac measures for Body Weight by Uncertainty. Panel A presents the log weighted memory error means from the store task. Panel B shows the cardiac deceleration score means from the information period. The arrow indicates the direction of greater cardiac deceleration.

overweight or obese subjects with low and high uncertainty. The relative deceleration of the low uncertainty group is shown both as a trend at the end of the pre period and also at the end of the information input period. Post-hoc tests on the mean difference scores demonstrated that, during low uncertainty input and initial storage overweight, subjects decelerated considerably more than underweight subjects. With high uncertainty input, however, overweight and underweight subjects did not differ in deceleration scores. The parallel between the deceleration and error scores should be noted. Within each weight group, relatively greater deceleration during initial information input and storage was associated with relatively fewer memory errors during the subsequent store task. Figure 3 illustrates this parallel. In Panel A, the error score interaction between body weight and uncertainty is presented; in Panel B, the comparable deceleration score interaction from the information input period is presented.

\section{DISCUSSION}

\section{Cognitive processes and cardiac change}

In an attempt to extend the Lacey hypothesis, the current experiment related cardiac changes to two alternative dimensions of cognitive processing: cognitive manipulation and memory load. Although the cognitive tasks employed had clear effects on heart rate, these effects were not related in a straightforward one-to-one fashion to demands for either cognitive manipulation or memory. Among the tasks assumed to involve significant cognitive manipulation, the two add tasks produced acceleration, but the rule discovery task produced deceleration. The relative absence of acceleration in the store task questioned the memory-load hypothesis. Alternative expectations would have predicted a relationship between difficulty or energy-demand ratings and cardiac acceleration. This relationship did not occur in any straightforward fashion either. The add task, for example, was rated low in difficulty and energy demand, but significant acceleration occurred on this task.

A set of regression analyses, however, provided evidence of the relative strength of the association of memory and rated energy demands with cardiac change. These analyses investigated relationships within individuals and provided a quantitative estimate of degree of relationship between tasks and cardiac responses. Rankings based on memory load and energy demand were related significantly to both accelerative and decelerative changes. Cognitive manipulation ranks were not related as strongly as those for memory load and energy demand. In addition, other aspects of the results were also consistent with a relation between memory requirements and cardiac change: (a) memory load was consistently related to absence of deceleration and often related to acceleration (post-hoc test results, and (b) memorizing during the information period seemed to be associated with stable acceleration.

With regard to the latter observation, a cardiac decleration during the input of the six numbers had been expected on the basis of the original Lacey sensory intake hypothesis. The potential influence of memorizing during this period had been disregarded due to the demonstrated robustness of the deceleration phenomenon in the face of situations involving such known cardiac accelerators as electric shock and physical exercise (Chase, Graham, \& Graham, 1968; Obrist, 1968). In fact, however, the dominant reaction during the information period in the present experiment was a strong cardiac acceleration instead of deceleration. In retrospect, this finding is not surprising. Kahneman, Tursky, Shapiro, and Crider (1969) found similar acceleration when subjects were listening to numbers and, within $2 \mathrm{sec}$, adding a constant integer to them. If the addition task was delayed $5 \mathrm{sec}$, however, cardiac deceleration was found during the input of numbers (Tursky, Schwartz, \& Crider, 1970). Thus, input of information with roughly concurrent demands for memorizing seems to produce cardiac acceleration.

The present emphasis placed upon memory load and memorizing is somewhat novel. A number of studies reviewed by Elliott (1972) and Hahn (1973) relate cardiac changes to either Lacey's sensory intake-rejection dimension or to arousal-type variables. These studies, as well as those supporting Lacey's association of cardiac acceleration with "mental elaboration," may be interpreted in terms of memory load (Elliott, 1972; Hahn, 1973; Kahneman et al., 1969; Lacey et al., 1963; Lacey, B., \& Lacey, J., 1974; Lacey, J., \& Lacey, B., 1974; Tursky et al., 1970; Jennings, 1971). Most tasks studied have required a combination memory, cognitive, and energy requirements.

Energy demands as well as memory requirements were related by the regression analyses to cardiac changes during information processing. Particularly in the 
absence of clear-cut results in the mean cardiac data, it would be unwise to impute the psychological control of heart rate to a single factor. Energy and memory requirements are not viewed as opposed notions but as interacting aspects of the processing task. Memory requirements may create, in part, the perceived energy requirements. The difficult empirical tasks facing investigators are, first, to independently measure energetic and cognitive variables and, second, to determine their joint relation to physiological variables (cf. Elliott, 1972; Kahneman, 1973; Lacey, J., \& Lacey, B., 1974). The current results suggest that studies of information processing with significant memory loads may be fruitful in this regard.

\section{Body Weight and the Schachter Hypothesis}

Some evidence for "sensory binding" in the overweight was found but such evidence was dependent upon input uncertainty. Evidence for sensory binding would have been obtained if overweight subjects had performed relatively better on tasks which did not combine a visual display with a processing requirement. Despite some trends, clear evidence on this point was not found. If sensory binding in fact occurred, the overweight might have been expected to show generally greater cardiac deceleration than the underweight. Although cardiac decelerative changes were not marked in general, overweight subjects showed larger decelerations than the underweight subjects in both the information input and task performance periods.

High uncertainty input produced an unexpected reversal in the direction of the performance and cardiac results. Relative to the underweight, the overweight showed more errors in the store task and cardiac deceleration was absent. Thus, the results for low uncertainty input provided limited support for Schachter's hypothesis, but the results for high uncertainty input contradict a simple sensory binding view. By increasing problem difficulty (and thus errors), high uncertainty input may have altered the processing strategy or heightened the emotional state of obese (cf. Nisbett, 1972; Singh, Swanson, Letz, \& Sanders, 1973). These results suggest that characteristics of stimulus material and processing performed must be taken into account within any "sensory binding" hypothesis.

Two studies of nonfood related information processing in the obese (Rodin, Herman, \& Schachter, cited in Rodin, 1973; and Rodin, 1973) show general agreement with the current results. The overweight performed better than normal subjects on simple memory tasks, disjunctive reaction times, and proofreading. With distracting emotional auditory input, however, the performance of the obese deteriorated more than the normals (Rodin, 1973). Less emotional auditory input did not prove distracting. The failure to find distraction with nonemotional input also questions a simple sensory-binding view.
In summary, the results suggest that changes in heart rate may be related to relatively specific aspects of cognitive tasks and to individual differences in information processing. Cardiac responses to the different tasks were related both to memory and energy demands. Suggestive evidence from cardiac and performance results indicated that sensory-binding effects in the obese may be dependent on the characteristics of the stimuli presented.

\section{REFERENCES}

Chase, W. G., Graham, F. D., \& Graham, D. T. Components of the heart rate response in anticipation of reaction time, and exercise tasks. Journal of Experimental Psychology, 1968, 76, 642-648.

Cicchetti, D. V. Extension of multiple-range tests to interaction tables in the analysis of variance: $A$ rapid approximate solution. Psychological Bulletin, 1972, 77, 405-408.

Davison, M. L. Univariate versus multivariate tests in repeatedmeasures experiments. Psychological Bulletin, 1972, 77, 446-452.

Elliot, R. The significance of heart rate for behavior: A critique of Lacey's hypothesis. Journal of Personality and Social Psychology, 1972, 22, 398-409.

HaHn, W. W. Attention and heart rate: A critical appraisal of the hypothesis of Lacey and Lacey. Psychological Bulletin, 1973, 79, 59-70.

Jennings, J. R. Cardiac reactions and different developmental levels of cognitive functioning. Psychophysiology, $1971,8,433-450$.

Jennings, J. R., Stringfellow, J. C., \& Graham, M. A comparison of the statistical distributions of beatby-beat heart rate and heart period. Psychophysiology, 1974, 11, 207-210.

Kahneman, D. Attention and effort. Englewood Cliffs, New Jersey: Prentice-Hall, 1973.

Kahneman, D., Tursky, B., Shapiro, D., \& Crider, A. Pupillary, heart rate, and skin resistance changes during a mental task. Journal of Experimental Psychology, 1969, 79, 165-167.

Khachturian, Z. S., Kerr, J., Kruger, R., \& Schachter, J. A methodological note: Comparison between period and rate data in studies of cardiac function. Psychophysiology, 1972, 9, 539-545.

LACEY, B. C., \& LACEY, J. I. Studies of heart rate and other bodily processes in sensorimotor behavior. In P. A. Obrist, A. Black, J. Brener, \& L. DiCara (Eds.), Cardiovascular psychophysiology-Current issues in response mechanisms, biofeedback and methodology. Chicago, Ill: Aldine-Atherton, 1974.

LACEY, J. I. Somatic response patterning and stress: Some revisions of activation theory. In M. H. Appley \& R. Trumbull (Eds.), Psychological stress: Issue in research. New York: Appleton-Century-Crofts, 1967. Pp. 14-42.

Lacey, J. I., Kagan, J., Lacey, B. C., \& Moss, H. A. The visceral level: Situational determinants and behavioral correlates of autonomic response patterns. In P. H. Knapp (Ed.), Expression of the emotions in man. New York: International Universities Press, 1963. Pp. 161-196. 
Metropolitan Life Insurance Company. New weight standards for men and women. Statistical Bulletin, 1959, 40 $1-4$.

Nisbett, R. E. Hunger, obesity, and the ventromedial hypothalamus. Psychological Review, 1972, 79, 433-453.

Ов RIST, P. A. Heart rate and somatic-motor coupling during classical aversive conditioning in humans. Journal of Experimental Psychology, 1968, 77, 180-193.

Rodin, J. Effects of distraction on performance of obese and normal subjects. Journal of Comparative and Physiological Psychology, 1973, 83, 68-75.

SCHACHTER, S. Some extraordinary facts about obese humans and rats. American Psychologist, 1971, 26, 129-144.

Singh, D., Swanson, J., Letz, R., \& Sanders, M. K. Performance of obese humans on transfer of training and reaction time tests. Psychosomatic Medicine, 1973, 35, 240249.

Tursky, B., Schwartz, G. E., \& Crider, A. Differential patterns of heart rate and skin resistance during a digittransformation task. Journal of Experimental Psychology, 1970, 83, 451-457.

WINER, B. J. Statistical principles in experimental design. New York: McGraw-Hill, 1962.

\section{NOTES}

1. For the first 24 subjects, the button press was not required for two tasks (read and store). Results of the first 24 subjects did not differ, however, substantively from those of the remaining subjects.

2. Alternative difference scores assessing acceleration and deceleration can be based on change from pre period maximum and minimum instead of the pre period mean. The latter was used because it is a more stable estimate of initial level than the maximum or minimum. In addition, the pre period maximum in the current experiment reflected the deceleration prior to task onset noted earlier. For completeness, however, statistical analysis analogous to those to be reported were performed on scores formed using the pre period maximum and minimum. The pattern of results was substantially the same as that for the difference scores reported.

3. The memory error score was created by scoring errors of omission 2 and errors of order 1 . The resulting scores showed a skewed distribution. Therefore, the logs of the values were used as the memory scores.

(Received for publication March 3, 1975; revision accepted April 25, 1974.) 